\title{
Thoracic Ultrasound-Assisted Selection for Pleural Biopsy With Abrams Needle
}

\author{
Maribel Botana-Rial MD PhD, Virginia Leiro-Fernández MD PhD, \\ Cristina Represas-Represas MD, Ana González-Piñeiro MD, \\ Amara Tilve-Gómez MD, and Alberto Fernández-Villar MD PhD
}

\begin{abstract}
BACKGROUND: Closed pleural biopsy (CPB) in patients with malignant pleural effusion is less sensitive than cytology. Ultrasound-assisted CPB allows biopsies to be performed in the lower thoracic parietal pleura, where secondary spread from pleural metastases is initially more likely to be found. We analyzed whether choosing the point of entry for CPB with thoracic ultrasound assistance influences the diagnostic yield in malignant pleural effusion. METHODS: This prospective study included patients who underwent CPB performed by an experienced pulmonologist in 2008-2010 (group A) and thoracic ultrasound was used to select the biopsy site. The results were compared with a historical series of CPB performed by the same pulmonologist without the assistance of thoracic ultrasound (group B). An Abrams needle was used in all cases. We analyzed the obtaining of pleural tissue and the diagnostic yield. RESULTS: We included 114 CPBs from group A (23\% tuberculous pleural effusion, $27 \%$ malignant pleural effusion) and 67 CPBs from group B $(24 \%$ tuberculous pleural effusion, $30 \%$ malignant pleural effusion) $(P=.70)$. Pleural tissue was obtained in $96.5 \%$ of the group A CPBs and $89.6 \%$ of the group B CPBs $(P=.05)$. The diagnostic yields of CPB for tuberculous pleural effusion and malignant pleural effusion in group $A$ were $89.5 \%$ and $77.4 \%$, respectively, and $91.7 \%$ and $60 \%$, respectively, in group $B(P=.80$ for tuberculous pleural effusion, and $P=.18$ for malignant pleural effusion). CONCLUSIONS: Selecting the point of entry for CPB using thoracic ultrasound increases the likelihood of obtaining pleural tissue and the diagnostic yield, but without statistical significance. We recommend ultrasound-assisted CPB to investigate pleural effusion, since the diagnostic yield of a pleural biopsy with an Abrams needle increased by $>\mathbf{1 7 \%}$ in subjects with malignant pleural effusion. Key words: Abrams pleural needle; biopsy pleural; diagnostic; malignant pleural effusion; thoracic ultrasound. [Respir Care 2013;58(11):1949-1954. (C) 2013 Daedalus Enterprises]
\end{abstract}

\section{Introduction}

Pleural effusion can occur as a complication of more than 50 different diseases, and accurately establishing a

Drs Botana-Rial, Leiro-Fernández, Represas-Represas, and FernándezVillar are affiliated with the Bronchopleural Unit, Department of Pneumology; Dr González-Piñeiro is affiliated with the Department of Pathology; and Dr Tilve-Gómez is affiliated with the Department of Radiology, Complexo Hospitalario Universitario de Vigo, Vigo, Spain.

The authors have disclosed no conflicts of interest.

Correspondence: Alberto Fernández-Villar MD PhD, Pneumology Service, Complexo Hospitalario Universitario de Vigo, Rúa Pizarro 22, 36204, Vigo, Spain. E-mail: alberto.fernandez.villar@sergas.es.

DOI: $10.4187 /$ respcare. 02378 diagnosis is an essential step in the management of these patients. After parapneumonic pleural effusion, the most frequent causes of exudative pleural effusion are malignant pleural effusion and tuberculous pleural effusion. ${ }^{1-3}$ To differentiate among the various causes of exudative pleural effusion, biochemical, microbiological, and cytological analyses of the pleural liquid should be performed. ${ }^{1,2}$ However, after an initial thoracocentesis, approximately $40 \%$ of patients remain undiagnosed; thus, the next step is a pleural biopsy. ${ }^{1,2}$ Some controversy exists about the ideal procedure that should be used to perform a pleural biopsy. ${ }^{4}$ The pleural biopsy can be a closed pleural biopsy (CPB), pleural biopsy guided by imaging, or thoracoscopy..$^{1,2}$

The diagnostic yield of CPB with respect to malignant pleural effusion is lower than that of cytology, with the 
reported sensitivities ranging from $48 \%-56 \%$ for CPB. ${ }^{1-3}$ One possible reason for the low yield could be patchy involvement of the pleura in malignant pleural effusion. This low yield does not occur in tuberculous pleural effusion, which has a diffuse expression throughout the pleural surfaces, and for which sensitivity can reach $85 \% .^{1,2}$ Another factor that might influence the diagnostic yield of $\mathrm{CPB}$ is that pleural malignant deposits tend to predominate close to the midline and diaphragm, which are areas that are best avoided when performing CPB.${ }^{4}$ Based on its low diagnostic sensitivity, the utility of $\mathrm{CPB}$ in the investigation of exudative pleural effusion has been questioned. ${ }^{4,5}$

Thoracoscopy is the gold standard for the diagnosis of malignant pleural effusion. ${ }^{2,3}$ Nevertheless, some studies have proposed that $\mathrm{CPB}$ guided by imaging can obtain a diagnostic yield similar to that of thoracoscopy.$^{6-10}$ Pleural biopsy guided by imaging (computed tomography [CT] or ultrasound scan) or thoracoscopy should be performed in almost all patients with exudative pleural effusion of unknown etiology. 2,11

Ultrasound-assisted CPB allows biopsies to be performed in the lower thoracic parietal pleura, where the secondary spread from pleural metastases is more likely to be initially found, and may lead to improved diagnostic yield. ${ }^{3,5}$ Recently, Koegelenberg et al, in a study performed with another objective, determined that an ultrasound-assisted Abrams pleural needle had a higher diagnostic yield than had been previously reported. ${ }^{12}$ The authors hypothesized that this high yield might be due to the use of ultrasound, which enables the biopsy to be performed closer to the diaphragm. ${ }^{12}$ Nevertheless, to the best of our knowledge, clear evidence supporting the use of ultrasound to select the lower thoracic parietal pleura as an appropriate biopsy site does not currently exist. The aim of the present study was to analyze whether selecting the point of entry of the CPB using ultrasound influences the diagnostic yield in patients with malignant pleural effusion independently of the presence of thickened or diseased pleura.

\section{Methods}

The local ethics committee approved this study, and all subjects provided informed consent.

\section{Subjects}

All patients who underwent CPB for exudative pleural effusion at the Unit of Interventional Bronchopleural Pathology in the Pneumology Department of the University Hospital Complex of Vigo were included in this study. All of the CPBs were performed by the same pulmonologist with extensive experience in the management of pleural disease or by a fellow in training (supervised by the expert), both of whom followed the recommendations of

\section{QUICK LOOK}

\section{Current knowledge}

In patients with malignant pleural effusion, closed pleural biopsy is less sensitive than cytology. Ultrasoundassisted closed pleural biopsy allows biopsy of the lower thoracic parietal pleura, where secondary spread from pleural metastases is more likely to be found.

\section{What this paper contributes to our knowledge}

Selecting the biopsy point of entry with ultrasound increased the likelihood of obtaining pleural tissue and the diagnostic yield by $>17 \%$, but the difference was not statistically significant.

published guidelines. ${ }^{1,2,13}$ In all cases at least one sample of pleural specimen was obtained. Between 2008 and 2010 a biopsy site in the lower region was selected by thoracic ultrasound. The results were compared with a historical series of CPBs that were performed without the assistance of thoracic ultrasound between 2005 and 2007; in this series, the entry point was chosen based on physical examination (percussion and auscultation). ${ }^{13}$

\section{Approach to Pleural Biopsy}

An initial diagnostic thoracocentesis for biochemical, microbiological, and cytological studies was performed in all subjects. If a diagnosis was not obtained after this test, a second cytology and a CPB with an Abrams needle were performed in subjects with exudative pleural effusion. The technique used for CPB was similar, and the Abrams needle was always used. The CPB was performed with the subject sitting with his or her back vertical and with the arm of the side containing the fluid placed over the opposite shoulder. Between 2005 and 2007, the entry point was chosen based on physical examination (percussion and auscultation). In the other group (2008-2010) thoracic ultrasound was used to locate the lower point of entry.

After the site was selected, the skin was cleaned and local anesthesia administered ( $2 \%$ mepivacaine). At least 4 separate biopsy specimens were obtained, placed in formalin, and taken to the pathology department; one specimen was placed in a sterile tube and sent to be cultured for mycobacteria. A chest radiograph was obtained after the CPB to verify that no pneumothorax had occurred.

In some subjects, in whom the cause of pleural effusion had still not been identified with CPB, a thoracoscopy was conducted; in others a clinical and radiological follow-up of at least 1 year were undertaken to demonstrate no symptoms or recurrence of pleural effusion. ${ }^{14}$ The decision to 
use one option or the other depended on the subject's pulmonologist.

The etiology of the pleural effusion was determined based on accepted criteria, as described by the Society of Spanish Pneumology and Thoracic Surgery. ${ }^{1,14}$ The clinical and epidemiological characteristics were recorded. For classification of the radiological characteristics of the pleural effusion, the following criteria were used:

- Size: classified as pleural effusion more than two thirds when it produced opacification of the entire hemithorax or the fluid reached the arch of the aorta, and as pleural effusion less than two thirds when it did not reach this threshold.

- Distribution: classified as free pleural effusion when it was mobile in the lateral decubitus on the chest radiograph, and as loculated when it was immobile.

- Presence or absence of pleural thickening $>5 \mathrm{~mm}$ revealed by $\mathrm{CT}$.

- With respect to the results of the CPB, an adequate pleural biopsy specimen was defined as evidence of pleural tissue in the sample. Tuberculous pleural effusion was diagnosed if a microbiology smear or cultures from the pleural tissue were positive for acid-fast bacilli, or if noncaseating granulomas were present in subjects with lymphocytic predominant effusion who responded to anti-tuberculosis therapy and who did not have other pleural granulomatous diseases. The presence of malignant cells in the pleural tissue established cancer as the etiology. Complications of CPB were registered in both series.

\section{Statistical Analysis}

The results are reported as number and percent for qualitative variables, and as mean \pm SD for quantitative variables. The Fisher exact test was used to compare qualitative variables. The analyses were performed with statistics software (SPSS 14.0, SPSS, Chicago, Illinois).

\section{Results}

\section{Subjects}

A total of 181 subjects underwent CPB during the study period. Of these, 114 subjects underwent ultrasound-assisted CPB between 2008 and 2010, and 67 subjects underwent CPB without ultrasound-assistance between 2005 and 2007 (control group). The mean \pm SD age of the subjects was $61.5 \pm 19 \mathrm{y}$, and $61.9 \%$ of the subjects were men. The etiologies of the pleural effusion are listed in Table $1(P=.70)$. Diagnostic thoracoscopies were performed in $13(11.4 \%)$ subjects in the ultrasound group, and
Table 1. Etiology of Pleural Effusion in Patients Who Underwent Closed Pleural Biopsy or Ultrasound-Assisted Closed Pleural Biopsy

\begin{tabular}{|c|c|c|}
\hline & $\begin{array}{l}\text { Closed Pleural } \\
\text { Biopsy } \\
n=67 \\
\text { no. }(\%)\end{array}$ & $\begin{array}{c}\text { Ultrasound-Assisted } \\
\text { Closed Pleural } \\
\text { Biopsy } \\
n=114 \\
\text { no. }(\%)\end{array}$ \\
\hline Tuberculous pleural effusion & $16(23.9)^{*}$ & $26(22.8) \dagger$ \\
\hline Malignant pleural effusion & $20(29.9)$ & $31(27.2)$ \\
\hline Parapneumonic pleural effusion & $2(3)$ & $1(0.9)$ \\
\hline \multicolumn{3}{|l|}{ Non-malignant pleural effusion $\ddagger$} \\
\hline Idiopathic pleural effusion & $6(9)$ & $13(11.4)$ \\
\hline Reactive pleural effusion & $4(6)$ & $7(6.1)$ \\
\hline Transudative & $1(1.5)$ & $3(2.7)$ \\
\hline Miscellaneous & $5(7.5) \S$ & $6(5.2) \|$ \\
\hline Not diagnostic & $13(19.4)$ & $27(23.7)$ \\
\hline \multicolumn{3}{|c|}{$\begin{array}{l}\text { * Twelve patients had a positive stain or culture for Mycobacterium tuberculosis or typical } \\
\text { caseating granulomas in the pleural biopsy. The remainder were treated empirically for pleural } \\
\text { tuberculosis, without recurrence of the effusion. } \\
\dagger \text { Nineteen patients had a definitive diagnosis. The remainder were treated empirically, } \\
\text { without recurrence of the effusion. } \\
\ddagger \text { Nonmalignant pleural effusion of unknown origin in patients with any of: nonspecific } \\
\text { pleuritis observed at thoracoscopy, thoracotomy, or autopsy (idiopathic pleural effusion); or } \\
\text { absence of symptoms or recurrence of pleural effusion during the 1-year follow-up period } \\
\text { (reactive pleural effusion). } \\
\S \text { One patient }(1.5 \%) \text { with pericardial disease, } 2(3 \%) \text { with collagen vascular disease, } 1(1.5 \%) \\
\text { with subphrenic abscess, and } 1(1.5 \%) \text { with amyloidosis. } \\
\| \text { One patient }(0.9 \%) \text { with pericardial disease, } 1(0.9 \%) \text { with pleural effusion due to drug } \\
\text { reactions, } 1(0.9 \%) \text { with iatrogenic pleural effusion, } 2(1,8 \%) \text { with uremic pleuritis, and } \\
1(0.9 \%) \text { with pleural effusion due to collagen vascular disease. }\end{array}$} \\
\hline
\end{tabular}

in $6(9 \%)$ in the control group. Four $(6 \%)$ subjects in the control group and $7(6.1 \%)$ in the ultrasound group were followed up for $\geq 12$ months. These cases were deemed reactive pleural effusion.

In the ultrasound group, 27.2\% (31/114) had malignant pleural effusion, and $22.8 \%$ had tuberculous pleural effusion (19 with a microbiology smear or culture positive for acid-fast bacilli or the presence of noncaseating granulomas in the pleural tissue; the remainder responded to antituberculosis therapy, without recurrence of pleural effusion). In the control group, 29.9\% (20/67) had malignant pleural effusion and $23.9 \%$ (16/61) had tuberculous pleural effusion (12 subjects with a definitive diagnosis, and 4 who were treated empirically, without recurrence of pleural effusion).

In the control group 6 subjects had no chest radiograph and 6 had no CT. In the ultrasound group 5 subjects had no chest radiograph and 20 had no CT. There were no differences in terms of the radiological characteristics of the pleural effusions (Table 2).

\section{Diagnostic Yield and Diagnoses}

Adequate pleural biopsy specimens were obtained in $96.5 \%$ of the ultrasound subjects and $89.6 \%$ of the control 
Table 2. Radiological Characteristics of Pleural Effusions

\begin{tabular}{lccc}
\hline \hline & $\begin{array}{c}\text { Closed Pleural } \\
\text { Biopsy } \\
\text { no. (\%) }\end{array}$ & $\begin{array}{c}\text { Ultrasound-Assisted } \\
\text { Closed Pleural } \\
\text { Biopsy } \\
\text { no. (\%) }\end{array}$ & $P$ \\
\hline $\begin{array}{l}\text { Extent of pleural effusion } \\
\geq 2 / 3\end{array}$ & $17(25.4)$ & $30(26.3)$ & .90 \\
$\quad<2 / 3$ & $46(68.7)$ & $81(71.1)$ & \\
Distribution & & & \\
$\quad$ Free & $55(82.1)$ & $106(93)$ & .07 \\
$\quad$ Loculation & $6(9)$ & $3(2.6)$ & \\
Pleural thickening on & & & \\
$\quad$ computed tomography & & $15(13.2)$ & .40 \\
$\quad$ Yes & $7(10.4)$ & $79(69.3)$ & \\
$\quad$ No & $54(80.6)$ &
\end{tabular}

Table 3. Final Diagnoses of Malignant Pleural Effusions Biopsied With Abrams Pleural Needle

\begin{tabular}{lc}
\hline \hline \multicolumn{1}{c}{ Diagnosis } & no. $(\%)$ \\
\hline Closed pleural biopsy $(n=12)$ & \\
Lung adenocarcinoma & $4(33.3)$ \\
T-cell lymphoma & $2(16.6)$ \\
Mesothelioma & $1(8.3)$ \\
Gastrointestinal carcinoma & $1(8.3)$ \\
Metastatic adenocarcinoma & $1(8.3)$ \\
Lung squamous cell carcinoma & $1(8.3)$ \\
Leukemia & $1(8.3)$ \\
Ultrasound-assisted closed pleural biopsy $(n=24)$ & \\
Lung adenocarcinoma & $10(41.4)$ \\
Breast carcinoma & $5(20.8)$ \\
Metastatic adenocarcinoma & $2(8.3)$ \\
Other lung carcinomas & $2(8.3)$ \\
Mesothelioma & $1(4.2)$ \\
T-cell lymphoma & $1(4.2)$ \\
Lung squamous cell carcinoma & $1(4.2)$ \\
Ovarian carcinoma & $1(4.2)$ \\
Colon carcinoma & $1(4.2)$ \\
\hline
\end{tabular}

subjects $(P=.050)$. The diagnostic yield in the ultrasound group for tuberculous pleural effusion was $91.7 \%$ (17/19), compared with $89.5 \%(11 / 12)$ in the control group $(P=.80)$. In the subjects with malignant pleural effusion, ultrasoundassisted CPB was diagnostic in $77.4 \%$ (24/31), whereas CPB without ultrasound was diagnostic in $60 \%(12 / 20)$ $(P=.18)$. The diagnostic yield of the ultrasound-assisted Abrams needle increased by $>17 \%$ for malignant pleural effusion. The final diagnoses for the malignant pleural effusions are presented in Table 3.

\section{Complications}

There were no major complications (major hemorrhage, sepsis, or death). Pneumothorax occurred in 2.5\% (3/114) of the ultrasound subjects and $1.5 \%(1 / 67)$ of the control subjects $(P=.90)$. Only one subject (in the ultrasound group) required a pleural drainage system.

\section{Discussion}

Choosing the CPB entry point with the assistance of ultrasound nonsignificantly increased the acquisition of adequate pleural biopsy specimens, which should increase the diagnostic yield of malignant pleural effusion. One possible explanation for these results is that ultrasound enables biopsy of the lower thoracic parietal pleura, where the secondary spread from pleural metastases is more likely to be initially found. ${ }^{12,15}$

A pleural biopsy can be performed blind, image guided, or thoracoscopy guided. ${ }^{1,2,16-18}$ The optimal method for obtaining pleural tissue and a diagnosis in subjects with exudative pleural effusion of unknown etiology is controversial. ${ }^{4}$ An important benefit of thoracoscopy is the ability to obtain a diagnosis, drain the effusion, and perform a pleurodesis during the same procedure. ${ }^{17}$ However, thoracoscopy is more expensive and time consuming than other procedures. Various CPB needles have been described, including the Abrams, Cope, or Vim-Silverman needles, and cutting needle biopsy (eg, Tru-Cut). Several studies have revealed the importance of performing image-guided pleural biopsy with CT or ultrasound. Ultrasound should be considered, given its lower cost, safety profile, and simplicity. Image-guided pleural biopsy provides the highest diagnostic accuracy. ${ }^{6-9}$ Image-guided pleural biopsy with a Tru-Cut needle provides the highest sensitivity: $70 \%$ with ultrasound, $87 \%$ with CT. ${ }^{8,9}$ Especially in the presence of pleural masses, thickening or nodularity, CPB should be CT or ultrasound guided, and with a Tru-Cut needle. The Abrams needle is indicated in pleural effusion without pleural thickening or nodularity. ${ }^{4}$

Metintas et al found no significant differences in the sensitivities of medical thoracoscopy and CT-guided Abrams pleural needle biopsy in subjects with malignant pleural effusion. ${ }^{6}$ The diagnostic sensitivities were $94 \%$ and $87 \%$, respectively. However, the majority of those studies included subjects with pleural effusion and pleural thickening due to malignant mesothelioma. ${ }^{6-8,10}$

Image-guided CPB is the technique of choice, with a high diagnostic yield, in patients with masses or diffuse, nodular pleural thickening. $4,6,8,9,16$ Mesothelioma is the malignant pleural effusion included most frequently in CPB studies, but this does not reflect the patient population in the present study in our institution. ${ }^{6-8,10}$ The incidence of mesothelioma in our study was low, and image-guided pleural biopsy has the highest diagnostic accuracy for mesothelioma. ${ }^{10}$

The role of CPB in diagnosing malignant pleural effusion has been questioned because its diagnostic sensitivity 


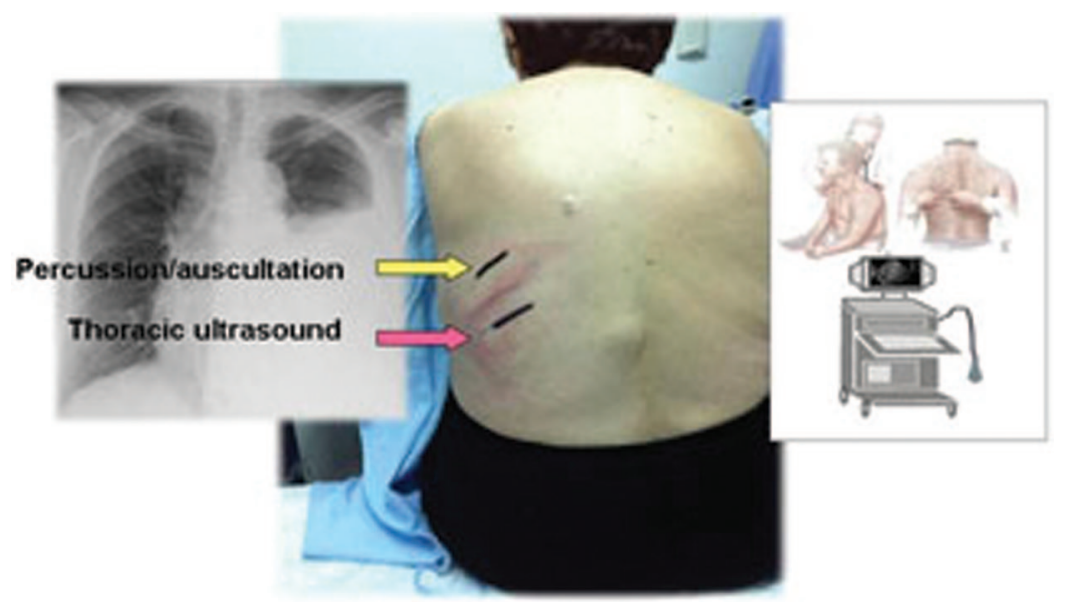

Figure. Choice of pleural biopsy entry point. Ultrasound enables biopsy in the lower thoracic parietal pleura, in contrast to biopsy guided by auscultation or percussion.

is less than that of image-guided and thoracoscopic pleural biopsy. ${ }^{4,5}$ For the diagnosis of pleural effusion with apparent diffuse pleura, such as that observed in tuberculous pleural effusion, CPB is the technique of choice. ${ }^{19}$ Ultrasound-guided pleural biopsy with an Abrams needle is more likely to contain pleural samples and has a significantly higher diagnostic sensitivity for pleural tuberculosis. ${ }^{12}$ But for patients with partially affected pleura or pleural thickening, such as in malignant pleural effusion, other techniques should be used. Another reason for the low diagnostic yield of CPB is that the pleural malignant deposits tend to be located predominantly near the midline and diaphragm, which are areas that should be avoided when performing an Abrams needle biopsy.,2,5 The direction of carcinomatous invasion is upward toward the costal pleura. Canto et al reviewed 203 diagnostic thoracoscopies in patients with malignant pleural effusion to determine the locations of the pleural metastases; $84 \%$ of the metastases were in the lower portions of the hemithorax, and only $53 \%$ of the patients had costal pleural involvement. ${ }^{15}$

In a study with 89 subjects, Koegelenberg et al found that ultrasound-assisted CPB performed with an Abrams needle had a significantly higher diagnostic sensitivity for tuberculous pleural effusion than did Tru-Cut needle biopsies. ${ }^{12}$ Malignant pleural effusion was diagnosed in 12 patients. The Abrams needle biopsies yielded histological confirmation in $83.3 \%$ of the samples. ${ }^{12}$ One possible explanation for the high yield is that the researchers chose low biopsy sites, such as the lower thoracic parietal pleura (close to the diaphragm), which are more likely to contain the secondary spread from visceral pleural metastases. Such an approach is possible with ultrasound assistance, but not with digital percussion as a guide. Unlike our study, there was a clinical suspicion of tuberculosis in the subjects in the Koegelenberg et al study, without an initial suspicion of malignant pleural effusion. In addition, the aim of their study was different. We believe that a thoracic ultrasound before ultrasound-assisted CPB would allow a lower (supra-diaphragmatic) biopsy site than can be selected with auscultation or percussion, which might improve the diagnostic yield of Abrams needle CPB for the diagnosis of malignant pleural effusion, as it did in our study (Figure).

The complication rates for image-guided pleural biopsy range from zero to $10 \%$. The risk of complications is lower than with blind biopsy. ${ }^{2,4,16,20}$ Although complications were not the primary focus of this study, serious complications, such as hemothorax or laceration of the spleen or kidney, did not occur in either group. The percentage of pneumothorax was higher in the ultrasound group, which might be in relation to the greater percentage of subjects with pleural effusion less than two thirds on chest radiograph.

To our knowledge, this study is the first direct comparison of the diagnostic yield of CPB and ultrasound-assisted Abrams needle biopsy in patients who were not selected with exudative pleural effusion, and with no differences in radiological characteristics or pleural thickening. A possible limitation of this study is that the increase in the acquisition of adequate pleural biopsy specimens should lead to a progressive increase in the experience of the operator. Nevertheless, the pleural biopsies were performed by an experienced physician who had completed more than 200 procedures before 2007, and the current evidence is that the diagnostic yield of the technique does not seem to depend greatly on the experience of the operator. ${ }^{18}$ In our study there were no significant differences in the characteristics of the 2 groups, but the diagnostic yield for malignant pleural effusion was $17 \%$ higher in the ultrasound group. 


\section{Thoracic Ultrasound-Assisted Selection for Pleural Biopsy With Abrams Needle}

\section{Conclusions}

Although CPB is less sensitive than thoracoscopy or image-guided pleural biopsy for the diagnosis of malignant pleural effusion, ultrasound assistance in Abrams needle CPB entry site nonsignificantly improved the acquisition of adequate pleural biopsy specimens, compared to site selection via digital percussion and auscultation, independently of the existence of masses or pleural thickening. Ultrasound-assisted CPB has a higher diagnostic sensitivity for malignant pleural effusion.

\section{REFERENCES}

1. Villena Garrido V, Ferrer Sancho J, Hernández Blasco L, de Pablo Gafas A, Pérez Rodríguez E, Rodríguez Panadero F, et al; Áreas de Técnicas y Trasplantes. SEPAR. [Diagnosis and treatment of pleural effusion]. Arch Bronconeumol 2006;42(7):349-372. Article in Spanish.

2. Hooper C, Lee YC, Maskell N. Investigation of a unilateral pleural effusion in adults: British Thoracic Society pleural disease guideline 2010. Thorax 2010;65(Suppl 2):ii4-ii17.

3. Roberts ME, Neville E, Berrisford RG, Antunes G, Ali NJ; BTS Pleural Disease Guideline Group. Management of a malignant pleural effusion: British Thoracic Society pleural disease guideline 2010. Thorax 2010;65(Suppl 2):ii32-ii40.

4. Koegelenberg CFN, Diacon AH. Pleural controversy: close needle pleural biopsy or thoracoscopy: which first? Respirology 2011;16(1): 738-746.

5. Ahrar K, Wallace M, Javadi S, Guptas S. Mediastinal, hilar, and pleural image-guided biopsy: current practice and techniques. Semin Respir Crit Care Med 2008;29(4):350-360.

6. Metintas M, Ak G, Dundar E, Yildirim H, Ozkan R, Kurt E, et al. Medical thoracoscopy vs CT scan-guided Abrams pleural needle biopsy for diagnosis of patiens with pleural effusions. Chest 2010; 137(6): 1362-1368.

7. Adams RF, Gleeson FV. Percutaneous image-guided cutting-needle biopsy of the pleura in the presence of a suspected malignant effusion. Radiology 2001;219(2):510-514.
8. Maskell NA, Gleeson FV, Davies RJ. Standard pleural biopsy versus CT-guided cutting-needle biopsy for diagnosis of malignant disease in pleural effusions: a randomised controlled trial. Lancet 2003; 361(9366):1326-1331.

9. Chang DB, Yang PC, Luh KT, Kuo SH, Yu CJ. Ultrasound-guided pleural biopsy with tru-cut needle. Chest 1991;100(5):1328-1333.

10. Adams RF, Gray W, Davies RJ, Gleeson FV. Percutaneous imageguided cutting needle biopsy of the pleura in the diagnosis of malignant mesothelioma. Chest 2001(6):120:1798-1802.

11. Janssen JP. Why you do or do not need thoracoscopy. Eur Respir Rev 2010;19(117):213-216.

12. Koegelenberg CFN, Bollinger CT, Theron J, Walzl G, Wright CA, Louw M, Diacon AH. A direct comparison of the diagnostic yield of ultrasound-assisted Abrams and tru-cut needle biopsies for pleural tuberculosis. Thorax 2010;65(10):857-862 .

13. Light RW, Lee YCG, editors. Textbook of pleural diseases, 2nd edition. London: Hodder Arnold; 2008.

14. Villena V, López-Encuentra A, Echave-Sustaeta J, Martín-Escribano P, Ortuño-de-Solo B, Estevez-Alfaro J. Diagnostic value of CA 549 in pleural fluid. Comparison with CEA, CA 15.3 and CA 72.4. Lung Cancer 2003;40(3):289-294.

15. Canto A, Rivas J, Saumench J, Morera R, Moya J. Points to consider when choosing a biopsy method in cases of pleurisy of unknown origin. Chest 1983;84(2):176-179.

16. Rahman NM, Gleeson FV. Image-guided pleural biopsy. Curr Opin Pulm Med 2008;14(4):331-336.

17. Rahman NM, Ali NJ, Brown G, Chapman SJ, Davies RJO, Downer NJ, Gleeson RV, et al. BTS pleural disease guideline group. Local anaesthetic thoracoscopy: British Thoracic Society pleural disease guideline 2010. Thorax 2010;65 (Suppl 2):ii54-ii60.

18. Chakrabarti B, Ryland I, Sheard J, Warburton CJ, Earis JE. The role of Abrams percutaneous pleural biopsy in the investigation of exudative pleural effusions. Chest 2006;129(6):1549-1555.

19. Diacon AH, Van de Wal BW, Wyser C, Smedema JP, Bezuidenhout J, Bollinger CT, Walzl G. Diagnostic tools in tuberculous pleurisy: a direct comparative study. Eur Respir J 2003;22(4):589-591.

20. Matin TN, Gleeson FV. Interventional radiology of pleural diseases. Respirology 2011;16(3):419-429. 